\title{
Studies on mass attenuation coefficient, effective atomic number and electron density of some amino acids
}

\author{
Turşucu $A^{1}$, Önder $P^{1}$, Demir $D^{1 \star}$ and Öznülüer $T^{2}$ \\ ${ }^{1}$ Department of Physics, Faculty of Science, Atatürk University, 25240 Erzurum, Turkey. \\ ${ }^{2}$ Department of Chemistry, Faculty of Science, Atatürk University, 25240 Erzurum, Turkey.
}

Accepted 29 January, 2013

\begin{abstract}
Mass attenuation coefficient $\left(\mu_{m}\right)$, effective atomic number $\left(Z_{e f f}\right)$ and effective electron density $\left(N_{e l}\right)$ were determined experimentally and theoretically for some amino acids (Glycine, L-Alanine, D-Alanine, Proline, L-Leucine and D-Leucine) at 8.04, 8.91, 13.37, 14.97, 17.44, 19.63, 22.10, 24.90, 30.82, 32.06, $35.40,36.39,37.26,43.74,44.48,50.38,51.70,53.16,80.99,276.40,302.85,356.01,383.85$ and $661.66 \mathrm{keV}$ photon energies by using an high-purity germanium (HPGe) detector with a resolution of $182 \mathrm{eV}$ at 5.9 $\mathrm{keV}$. The theoretical mass attenuation coefficients were estimated using mixture rule. The calculated values were compared with the experimental values for all amino acids. Good agreement has been observed between experimental and theoretical values within experimental uncertainties. The results show that mass attenuation coefficients, effective atomic numbers and effective electron densities depend on photon energy and chemical content. Also, the investigated parameters are different in different isomers (L- and D-) of the same molecule.
\end{abstract}

Key words: Mass attenuation coefficient, effective atomic number, effective electron density, high-purity germanium (HPGe) detector.

\section{INTRODUCTION}

In recent years, due to increasing use of medical physics and radiation biology, the study of photon-atom interaction parameters has attained a significant importance. These parameters are mass attenuation coefficient $\left(\mu_{m}\right)$, atomic and electronic cross-section, effective atomic number $\left(Z_{\text {eff }}\right)$ and effective electron density $\left(N_{e l}\right)$. Mass attenuation coefficient is a measure of the average number of interactions between incident photons and matter that occur in a given mass per unit area thickness of the substance under investigation (Jackson and Hawkes, 1981; Kerur et al., 1991). Mass attenuation coefficient tends to increase with increasing atomic number at the same photon energy, so materials with high atomic numbers (and, hence, high mass

\footnotetext{
${ }^{*}$ Corresponding author. E-mail: ddemir@atauni.edu.tr.
}

attenuation coefficients) are normally chosen to shield Xand $\mathrm{y}$ - radiation.

In photon interaction in the composite materials, the atomic number cannot be represented uniquely by a single number across the entire energy region, as in the case of pure elements. For composite materials this quantity is called the effective atomic number and it varies with energy (Hine, 1952). Effective atomic number is a convenient parameter for representing the attenuation of $X$ - and $\mathrm{Y}$ - rays in a complex medium and particularly for the calculation of the dose in radiation therapy. The energy absorption in a given medium can be calculated if certain constants are known. These necessary constants are effective atomic number and electron density of the medium. The effective atomic numbers for some chemical compounds containing $\mathrm{H}$-, Cand $\mathrm{O}$ - atoms were reported (Hiremath and Chikkur, 1993).

Biological molecules, such as carbohydrates, proteins, fats and oils, are performing a variety of functions in 
living systems. Amino acids are biochemical building blocks. They form short polymer chains called polypeptides or peptides which in turn form structures called proteins. The kinds of amino acids determine the shape of the proteins formed. Nineteen of the 20 amino acids used to synthesize proteins can exist as L- or Denantiomers. Enantiomers are two stereoisomers that are related to each other by a reflection; they are mirror images of each other, which are non-superimposable. Every stereogenic center in one has the opposite configuration in the other. L- and D-Alanine is the simplest chiral amino acid present in nature and is therefore an excellent model for understanding general structural and chemical properties of amino acids as seen from Figure 1. Two compounds that are enantiomers of each other have the same physical properties, except for the direction in which they rotate polarized light and how they interact with different optical isomers of other compounds. As a result, different enantiomers of a compound may have substantially different biological effects. Pure enantiomers also exhibit the phenomenon of optical activity and can be separated only with the use of a chiral agent (Voet and Voet, 1995). There are only few reports on mass attenuation coefficient, effective atomic number and electron density studies of amino acids in literature (Manohara and Hanagodimath, 2007a, b). Manohara and Hanagodimath (2007a, b), have reported the effective atomic numbers and electron densities of essential amino acids in the energy range 1 keV-100 GeV and $1 \mathrm{keV}$ to $20 \mathrm{MeV}$, respectively. Keszthelyi and Vincze (1975) have observed no difference in the absorption of right and left circulary polarized $\mathrm{y}$-radiation in L- and D-tryptophan and tyrosine with Mössbauer's (1975) method. There are reports on effective atomic number of several biologically important compounds in literature (Manjunathaguru and Umesh, 2006, 2009; Manohara et al., 2008a, b; Yang et al., 1987; Özyol, 1994; Rao et al., 1985; Gowda et al., 2005). Singh et al. (2010) have studied photon interaction and photon energy absorption effective atomic numbers for some amino acids. The molar extinction coefficients were determined in literature (Singh et al., 2004; Kumar et al., 2006). The effective atomic and electron numbers of some alloys were reported by Akkurt $(2007,2009)$.

In the present work, mass attenuation coefficients, effective atomic numbers and electron densities have been calculated for Glycine, L-Alanine, D-Alanine, Proline, L-Lueucine and D-Leucine at 8.04, 8.91, 13.37, 14.97, 17.44, 19.63, 22.10, 24.90, 30.82, 32.06, 35.40, $36.39,37.26,43.74,44.48,50.38,51.70,53.16,80.99$, $276.40,302.85,356.01,383.85$ and $661.66 \mathrm{keV}$ photon energies by using an high-purity germanium (HPGe) detector with a resolution of $182 \mathrm{eV}$ at $5.9 \mathrm{keV}$. Effective atomic numbers and electron densities have been calculated for these amino acids by using the measured mass attenuation coefficient values. Finally, the investigated parameters have been compared using the results of WinXCom program (Gerward et al., 2001).

\section{MATERIALS AND METHODS}

Mass attenuation coefficients for the different materials and energies can be determined by the radiation transmission. A narrow beam of mono-energetic photons with incident intensity $I_{0}$, penetrating a layer of material with mass thickness $t$ (the mass per unit area) and density $\rho$ emerges with intensity $I$ is given by the exponential attenuation law:

$$
I=I_{\mathrm{o}} \exp \left[-\mu_{m} t\right]
$$

Where $I / I_{0}$ is the transmission fraction $T$ and $\mu_{m}=\mu / \rho$ is the mass attenuation coefficient. The cross-section per molecule, $\sigma_{s}$, can be written in terms of an effective (average) cross-sections per atom, $\sigma_{a}$, and an effective (average) cross-section per electron, $\sigma_{e}$, as:

$\sigma_{s}=n \sigma_{a}=n Z_{e f f} \sigma_{e}$

Where $Z_{\text {eff }}$ is the effective atomic number and $n=\sum_{i} n_{i}$ is the total number of atoms present in a molecule. Essentially, it is assumed that the actual atoms of the molecule can be replaced by the same number of identical (average) atoms, each having $Z_{\text {eff }}$ electrons. $Z_{\text {eff }}$ is given by:

$Z_{\text {eff }}=\frac{\sum_{i} n_{i} A_{i}(\mu / \rho)_{i}}{\sum_{i} n_{i} A_{i} / Z_{i}(\mu / \rho)_{i}}$

Where $Z_{i}$ and $A_{i}$ are the atomic number and the atomic mass of the ith element present in a molecule, respectively. The effective electron density, expressed in the number of electrons per unit mass is closely related to the effective atomic number. The electron density can be generalized to a compound

$$
N_{e l}=N_{A} \frac{n Z_{e f f}}{\sum_{i} n_{i} A_{i}}=N_{A} \frac{Z_{e f f}}{\langle A\rangle}
$$

Where $N_{A}$ is the Avogadro constant and $\langle A\rangle$ is the average atomic mass of the compound. The theoretical $\mu_{m}$ values for present samples were obtained by WinXCom program. This program depends on applying the mixture rule to calculate the partial and total mass attenuation coefficients for all elements, compounds and mixtures at standard as well as selected energies.

The experimental setup used in the present study is shown in Figure 2. The source-sample and sample-detector distance was set to 30 and $30 \mathrm{~mm}$, respectively. Sharanabasappa et al. (2010) suggested the transmission range $0.5 \geq T \geq 0.02$ for the HPGe detectors. The samples of thickness ranging from 0.065 to 0.392 $\mathrm{g} / \mathrm{cm}^{2}$ were prepared in the form of $13 \mathrm{~mm}$ dia cylindrical pellets by pressing in a hand operated hydraulic press at a pressure of 8 ton. In order to irradiate the compounds at energies $30.82,35.40,53.16$, 
L-Alanine<smiles>C[C]([O-])[I-](C)[NH3+]</smiles>

D-Alanine<smiles>CC(C)([NH3+])C(=O)Cl</smiles>

Figure 1. The chemical structure of Alanine.

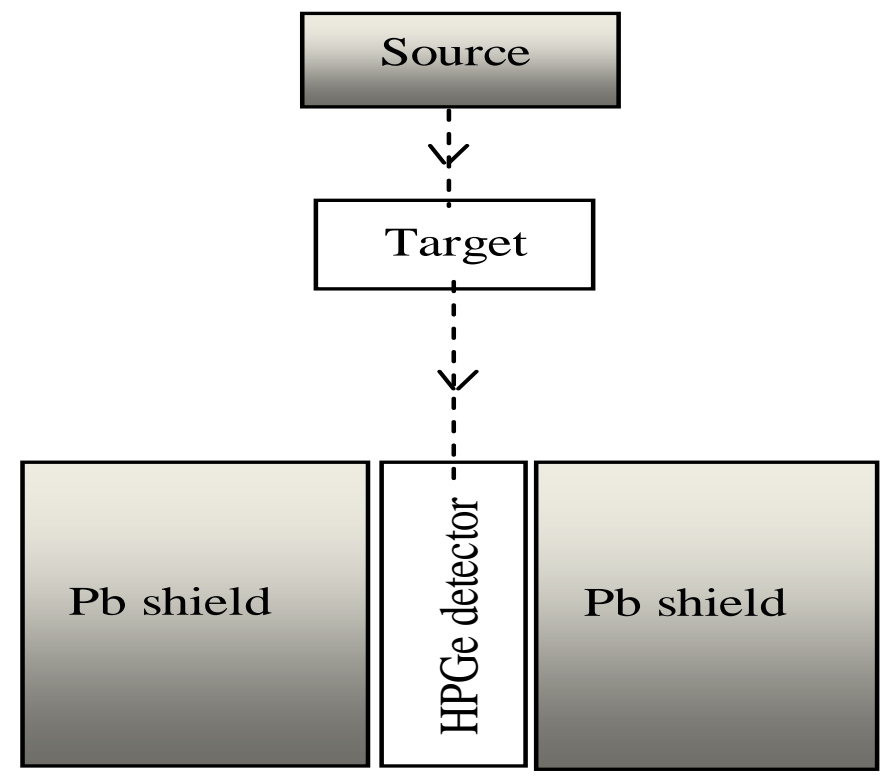

Figure 2. Experimental setup.

$80.99,276.40,302.85,356.01,383.85$ and $661.66 \mathrm{keV}$, polyester coated ${ }^{133} \mathrm{Ba}$ and ${ }^{137} \mathrm{Cs}$ radiation sources (intensity: $10 \mu \mathrm{Ci}$ ) were used. Also, we used a variable energy X-ray source from Amersham (AMC.2084) for energies 8.04, 8.91, 13.37, 14.97, $17.44,19.63,22.10,24.90,32.06,36.39,37.26,43.74,44.48,50.38$ and $51.70 \mathrm{keV}$. This source contains a sealed ceramic primary source $\left(\mathrm{Am}^{241}\right.$ of intensity $\left.10 \mathrm{mCi}\right)$ which excites characteristic $\mathrm{X}$ rays from six different targets ( $\mathrm{Cu}, \mathrm{Rb}, \mathrm{Mo}, \mathrm{Ag}, \mathrm{Ba}$ and $\mathrm{Tb}$ ) in turn (Figure 3).

Photon intensities were measured using the HPGe detector. The HPGe detector is a direct solar steam generation (DSG) planar HPGe crystal with a diameter of $16 \mathrm{~mm}$, a length of $10 \mathrm{~mm}$, a beryllium window of $0.12 \mathrm{~mm}$ and active area of $200 \mathrm{~mm}^{2}$. A bias voltage of $-1500 \mathrm{~V}$ is applied to the detector with a resolution of 182 $\mathrm{eV}$ at $5.9 \mathrm{keV}$. In this work, the studied amino acids were Glycine, L-Alanine, D-Alanine, Proline, L-Lueucine and D-Leucine. The spectra were recorded using a Canberra (AccuSpec) PC-based multichannel analyzer card. The time constant of the Ortec model 472 amplifier was set to $6 \mu \mathrm{s}$, ensuring optimum detector performance as specified by manufacturers. Operating parameters of the system are governed and controlled by the computer program Genie-2000. The pulse height spectra of X-rays were acquired for a period of 300 to $900 \mathrm{~s}$. The data were collected into 1024 channel of the MCA. Data were analyzed by the Origin 7.5 software program. The mean of ten channels of each side of the peaks was used to calculate the background and to define the peak. A linear background function was selected since the background is constant in this region. The background count rate was subtracted from measurements. $X$ - and $Y$-ray peaks have been fitted into one Gaussian. A typical spectrum of $\mathrm{Rb} K \mathrm{X}$-rays obtained with D-Leucine is shown in Figure 4.

\section{RESULTS AND DISCUSSION}

The chemical formulas and the mean atomic numbers of the amino acids studied in the present work are given in Table 1. The measured mass attenuation coefficients of amino acids at photon energies ranging from 8.04 to $661.66 \mathrm{keV}$ together with the theoretical values 


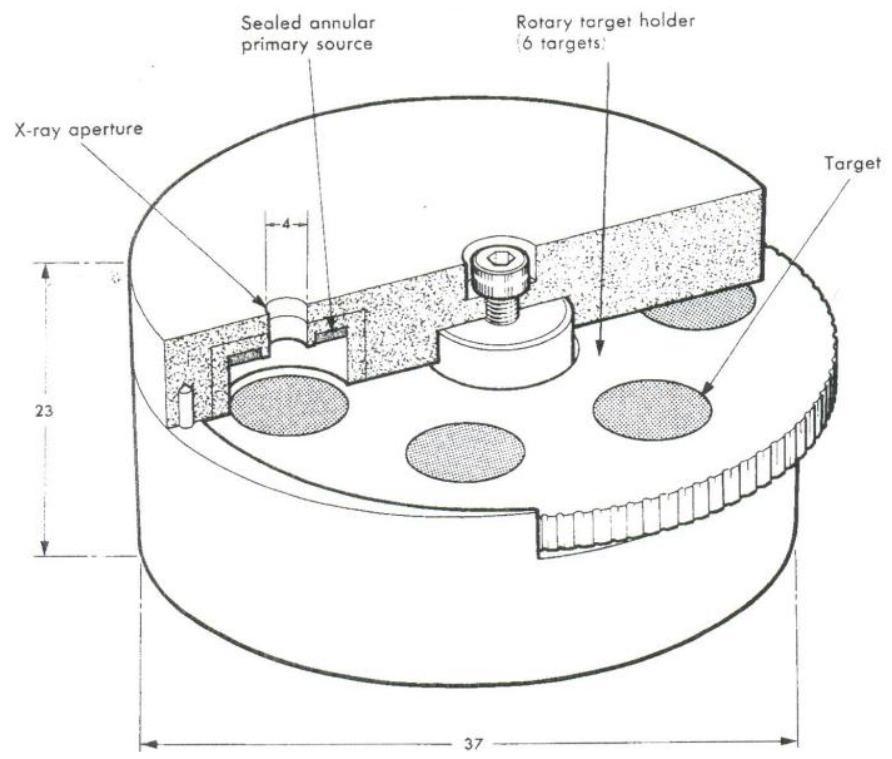

Figure 3. Variable energy X-ray source.

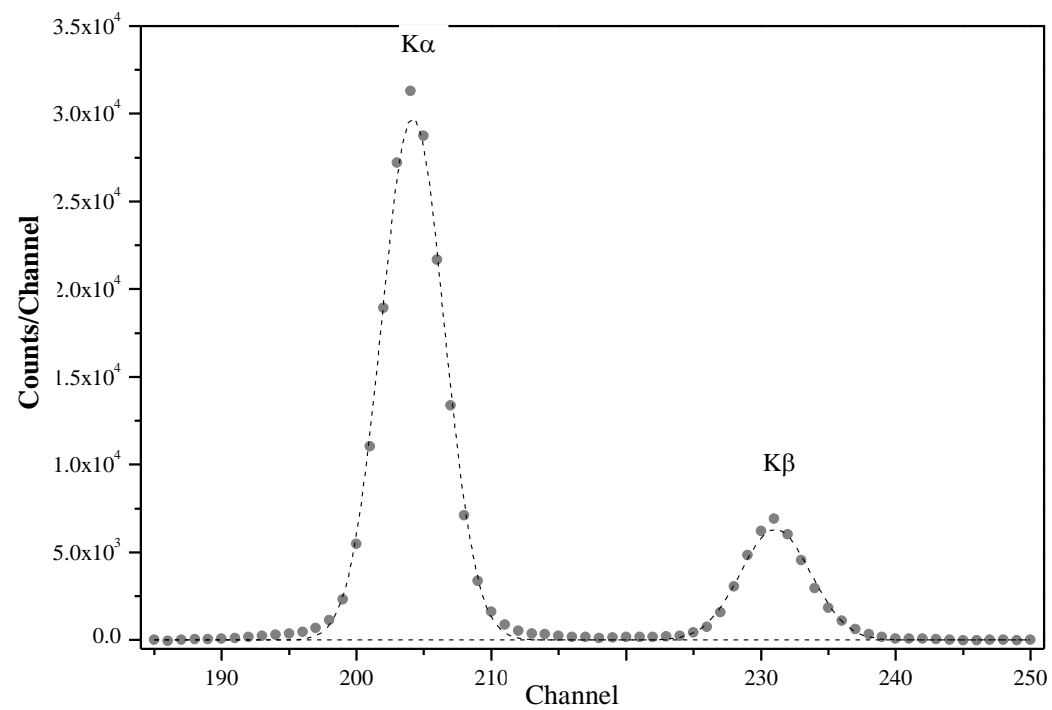

Figure 4. Spectrum of Rb K X-rays obtained with absorber (D-Leucine).

Table 1. The chemical formulas and the mean atomic numbers of some amino acids.

\begin{tabular}{lcc}
\hline Amino acid & Chemical formula & Mean atomic number, $\bar{Z}$ \\
\hline Glycine & $\mathrm{C}_{2} \mathrm{H}_{5} \mathrm{NO}_{2}$ & 4.00 \\
L-Alanine & $\mathrm{C}_{3} \mathrm{H}_{7} \mathrm{NO}_{2}$ & 3.69 \\
D-Alanine & $\mathrm{C}_{3} \mathrm{H}_{7} \mathrm{NO}_{2}$ & 3.69 \\
Proline & $\mathrm{C}_{5} \mathrm{H}_{9} \mathrm{NO}_{2}$ & 3.65 \\
L-Leucine & $\mathrm{C}_{6} \mathrm{H}_{13} \mathrm{NO}_{2}$ & 3.27 \\
D-Leucine & $\mathrm{C}_{6} \mathrm{H}_{13} \mathrm{NO}_{2}$ & 3.27 \\
\hline
\end{tabular}


Table 2. Mass attenuation coefficients $\left(\mathrm{cm}^{2} / \mathrm{g}\right)$ of some amino acids.

\begin{tabular}{|c|c|c|c|c|c|c|c|c|c|c|c|c|}
\hline \multicolumn{13}{|c|}{ Amino acids } \\
\hline $\begin{array}{c}\text { Energy } \\
(\mathrm{keV})\end{array}$ & $\begin{array}{c}\text { Glycine } \\
\text { experimental }\end{array}$ & Theoretical & $\begin{array}{c}\text { L-Alanine } \\
\text { experimental }\end{array}$ & Theoretical & $\begin{array}{c}\text { D-Alanine } \\
\text { experimental }\end{array}$ & Theoretical & $\begin{array}{c}\text { Proline } \\
\text { experimental }\end{array}$ & Theoretical & $\begin{array}{c}\text { L-Leucine } \\
\text { experimental }\end{array}$ & Theoretical & $\begin{array}{c}\text { D-Leucine } \\
\text { experimental }\end{array}$ & Theoretical \\
\hline 8.04 & $7.23 \pm 0.20$ & 7.74 & $7.13 \pm 0.20$ & 7.14 & $6.72 \pm 0.19$ & 7.14 & $6.75 \pm 0.19$ & 6.47 & $6.56 \pm 0.18$ & 6.11 & $5.88 \pm 0.16$ & 6.11 \\
\hline 8.91 & $5.21 \pm 0.14$ & 5.69 & $5.42 \pm 0.15$ & 5.25 & $4.83 \pm 0.13$ & 5.25 & $5.21 \pm 0.14$ & 4.77 & $4.44 \pm 0.12$ & 4.50 & $4.03 \pm 0.11$ & 4.50 \\
\hline 13.37 & $1.62 \pm 0.04$ & 1.77 & $1.55 \pm 0.04$ & 1.64 & $1.39 \pm 0.04$ & 1.64 & $1.77 \pm 0.05$ & 1.50 & $1.51 \pm 0.04$ & 1.43 & $1.29 \pm 0.04$ & 1.43 \\
\hline 14.97 & $1.21 \pm 0.03$ & 1.30 & $1.24 \pm 0.03$ & 1.22 & $1.02 \pm 0.03$ & 1.22 & $1.25 \pm 0.03$ & 1.12 & $1.11 \pm 0.03$ & 1.07 & $0.97 \pm 0.03$ & 1.07 \\
\hline 17.44 & $0.85 \pm 0.02$ & 0.89 & $0.75 \pm 0.02$ & 0.83 & $0.62 \pm 0.02$ & 0.83 & $0.69 \pm 0.02$ & 0.77 & $0.76 \pm 0.02$ & 0.74 & $0.60 \pm 0.02$ & 0.74 \\
\hline 19.63 & $0.68 \pm 0.02$ & 0.68 & $0.60 \pm 0.02$ & 0.64 & $0.50 \pm 0.02$ & 0.64 & $0.56 \pm 0.02$ & 0.60 & $0.60 \pm 0.02$ & 0.58 & $0.51 \pm 0.02$ & 0.58 \\
\hline 22.10 & $0.51 \pm 0.02$ & 0.53 & $0.51 \pm 0.02$ & 0.51 & $0.43 \pm 0.02$ & 0.51 & $0.44 \pm 0.02$ & 0.48 & $0.49 \pm 0.02$ & 0.46 & $0.40 \pm 0.02$ & 0.46 \\
\hline 24.90 & $0.40 \pm 0.02$ & 0.42 & $0.40 \pm 0.02$ & 0.41 & $0.33 \pm 0.02$ & 0.41 & $0.34 \pm 0.02$ & 0.39 & $0.35 \pm 0.02$ & 0.38 & $0.33 \pm 0.02$ & 0.38 \\
\hline 30.82 & $0.30 \pm 0.02$ & 0.31 & $0.32 \pm 0.02$ & 0.31 & $0.28 \pm 0.02$ & 0.31 & $0.30 \pm 0.02$ & 0.30 & $0.29 \pm 0.02$ & 0.29 & $0.26 \pm 0.02$ & 0.29 \\
\hline 32.06 & $0.31 \pm 0.02$ & 0.30 & $0.30 \pm 0.02$ & 0.29 & $0.26 \pm 0.02$ & 0.29 & $0.28 \pm 0.02$ & 0.28 & $0.29 \pm 0.02$ & 0.28 & $0.24 \pm 0.02$ & 0.28 \\
\hline 35.40 & $0.29 \pm 0.02$ & 0.27 & $0.29 \pm 0.02$ & 0.27 & $0.25 \pm 0.02$ & 0.27 & $0.26 \pm 0.02$ & 0.26 & $0.25 \pm 0.02$ & 0.26 & $0.24 \pm 0.02$ & 0.26 \\
\hline 36.39 & $0.25 \pm 0.02$ & 0.26 & $0.28 \pm 0.02$ & 0.26 & $0.22 \pm 0.02$ & 0.26 & $0.24 \pm 0.02$ & 0.25 & $0.25 \pm 0.02$ & 0.25 & $0.23 \pm 0.02$ & 0.25 \\
\hline 37.26 & $0.25 \pm 0.02$ & 0.26 & $0.24 \pm 0.02$ & 0.25 & $0.19 \pm 0.02$ & 0.25 & $0.20 \pm 0.02$ & 0.25 & $0.25 \pm 0.02$ & 0.25 & $0.22 \pm 0.02$ & 0.25 \\
\hline 43.74 & $0.20 \pm 0.02$ & 0.23 & $0.20 \pm 0.02$ & 0.23 & $0.18 \pm 0.01$ & 0.23 & $0.19 \pm 0.02$ & 0.22 & $0.22 \pm 0.02$ & 0.22 & $0.21 \pm 0.02$ & 0.22 \\
\hline 44.48 & $0.19 \pm 0.02$ & 0.23 & $0.18 \pm 0.01$ & 0.22 & $0.15 \pm 0.01$ & 0.22 & $0.19 \pm 0.02$ & 0.22 & $0.20 \pm 0.02$ & 0.21 & $0.19 \pm 0.02$ & 0.21 \\
\hline 50.38 & $0.18 \pm 0.01$ & 0.21 & $0.18 \pm 0.01$ & 0.21 & $0.15 \pm 0.01$ & 0.21 & $0.18 \pm 0.01$ & 0.21 & $0.20 \pm 0.02$ & 0.21 & $0.19 \pm 0.02$ & 0.21 \\
\hline 51.70 & $0.18 \pm 0.01$ & 0.21 & $0.15 \pm 0.01$ & 0.21 & $0.14 \pm 0.01$ & 0.21 & $0.17 \pm 0.01$ & 0.20 & $0.19 \pm 0.02$ & 0.21 & $0.17 \pm 0.01$ & 0.21 \\
\hline 53.16 & $0.16 \pm 0.01$ & 0.20 & $0.14 \pm 0.01$ & 0.20 & $0.13 \pm 0.01$ & 0.20 & $0.15 \pm 0.01$ & 0.20 & $0.18 \pm 0.02$ & 0.20 & $0.17 \pm 0.01$ & 0.20 \\
\hline 80.99 & $0.15 \pm 0.01$ & 0.17 & $0.14 \pm 0.01$ & 0.17 & $0.12 \pm 0.01$ & 0.17 & $0.13 \pm 0.01$ & 0.17 & $0.18 \pm 0.02$ & 0.18 & $0.17 \pm 0.01$ & 0.18 \\
\hline 276.40 & $0.13 \pm 0.01$ & 0.12 & $0.13 \pm 0.01$ & 0.12 & $0.12 \pm 0.01$ & 0.12 & $0.12 \pm 0.01$ & 0.12 & $0.14 \pm 0.01$ & 0.12 & $0.12 \pm 0.01$ & 0.12 \\
\hline 302.85 & $0.12 \pm 0.01$ & 0.11 & $0.13 \pm 0.01$ & 0.11 & $0.12 \pm 0.01$ & 0.11 & $0.11 \pm 0.01$ & 0.11 & $0.12 \pm 0.01$ & 0.12 & $0.11 \pm 0.01$ & 0.12 \\
\hline 356.01 & $0.11 \pm 0.01$ & 0.11 & $0.12 \pm 0.01$ & 0.11 & $0.11 \pm 0.01$ & 0.11 & $0.10 \pm 0.01$ & 0.11 & $0.11 \pm 0.01$ & 0.11 & $0.11 \pm 0.01$ & 0.11 \\
\hline 383.85 & $0.11 \pm 0.01$ & 0.10 & $0.11 \pm 0.01$ & 0.11 & $0.10 \pm 0.01$ & 0.11 & $0.10 \pm 0.01$ & 0.11 & $0.11 \pm 0.01$ & 0.11 & $0.10 \pm 0.01$ & 0.11 \\
\hline 661.66 & $0.07 \pm 0.01$ & 0.08 & $0.10 \pm 0.01$ & 0.08 & $0.09 \pm 0.01$ & 0.08 & $0.09 \pm 0.01$ & 0.08 & $0.10 \pm 0.01$ & 0.08 & $0.09 \pm 0.01$ & 0.08 \\
\hline
\end{tabular}

calculated using mixture rule from XCOM have are shown in Table 2. It is evident from the Table 2 that the measured values of this parameter were in good agreement with those obtained theoretically. It is clearly seen that mass attenuation coefficient depends on photon energy and chemical content. Mass attenuation coefficient values of the amino acids decrease with increasing photon energy.

Experimental as well as theoretical values of the effective atomic number are tabulated in Table 3. Good agreement has been achieved between measured and calculated values. As shown in Table 3, the present results clearly confirm the comment made by Hine (1952) mentioned earlier that the effective atomic number varies with energy.

The electron densities have been determined by using Equation 4 and mass attenuation coefficient values for the elements $\mathrm{H}-, \mathrm{C}_{-}, \mathrm{O}-$ and $\mathrm{N}$ -
Effective electron density values of the amino acids are listed in Table 4 . It is clearly seen that effective electron density depends on the photon energy and chemical content. The electron density is closely related to the effective atomic number and has the same qualitative energy dependence as effective atomic number.

The contribution of coherent (Rayleigh) scattering, incoherent (Compton) scattering and photoelectric process to total photon interaction 
Table 3. Effective atomic numbers of some amino acids.

\begin{tabular}{|c|c|c|c|c|c|c|c|c|c|c|c|c|}
\hline \multicolumn{13}{|c|}{ Amino acids } \\
\hline $\begin{array}{l}\text { Energy } \\
(\mathrm{keV})\end{array}$ & $\begin{array}{c}\text { Glycine } \\
\text { experimental }\end{array}$ & Theoretical & $\begin{array}{c}\text { L-Alanine } \\
\text { experimental }\end{array}$ & Theoretical & $\begin{array}{c}\text { D-Alanine } \\
\text { experimental }\end{array}$ & Theoretical & $\begin{array}{c}\text { Proline } \\
\text { experimental }\end{array}$ & Theoretical & $\begin{array}{c}\text { L-Leucine } \\
\text { experimental }\end{array}$ & Theoretical & $\begin{array}{c}\text { D-Leucine } \\
\text { experimental }\end{array}$ & Theoretical \\
\hline 8.04 & $6.71 \pm 0.21$ & 7.18 & $7.00 \pm 0.22$ & 7.01 & $6.60 \pm 0.21$ & 7.01 & $7.09 \pm 0.23$ & 6.79 & $7.16 \pm 0.23$ & 6.67 & $6.42 \pm 0.21$ & 6.67 \\
\hline 8.91 & $6.54 \pm 0.21$ & 7.14 & $7.18 \pm 0.23$ & 6.96 & $6.40 \pm 0.20$ & 6.96 & $7.37 \pm 0.24$ & 6.75 & $6.50 \pm 0.21$ & 6.59 & $5.90 \pm 0.19$ & 6.59 \\
\hline 13.37 & $6.16 \pm 0.20$ & 6.74 & $6.10 \pm 0.20$ & 6.45 & $5.47 \pm 0.18$ & 6.45 & $7.36 \pm 0.24$ & 6.23 & $6.32 \pm 0.20$ & 5.98 & $5.40 \pm 0.17$ & 5.98 \\
\hline 14.97 & $6.05 \pm 0.19$ & 6.50 & $6.35 \pm 0.20$ & 6.25 & $5.22 \pm 0.17$ & 6.25 & $6.72 \pm 0.22$ & 6.02 & $5.93 \pm 0.19$ & 5.72 & $5.18 \pm 0.17$ & 5.72 \\
\hline 17.44 & $5.93 \pm 0.19$ & 6.21 & $5.28 \pm 0.17$ & 5.88 & $4.36 \pm 0.14$ & 5.88 & $5.05 \pm 0.16$ & 5.64 & $5.43 \pm 0.17$ & 5.30 & $4.28 \pm 0.14$ & 5.30 \\
\hline 19.63 & $5.93 \pm 0.19$ & 5.93 & $5.21 \pm 0.17$ & 5.56 & $4.34 \pm 0.14$ & 5.56 & $5.02 \pm 0.16$ & 5.38 & $5.16 \pm 0.17$ & 4.97 & $4.39 \pm 0.14$ & 4.97 \\
\hline 22.10 & $5.40 \pm 0.17$ & 5.61 & $5.30 \pm 0.17$ & 5.24 & $4.47 \pm 0.14$ & 5.24 & $4.68 \pm 0.15$ & 5.11 & $4.93 \pm 0.16$ & 4.66 & $4.02 \pm 0.13$ & 4.66 \\
\hline 24.90 & $5.01 \pm 0.16$ & 5.26 & $4.84 \pm 0.16$ & 4.94 & $4.00 \pm 0.13$ & 4.94 & $4.19 \pm 0.13$ & 4.80 & $4.01 \pm 0.13$ & 4.36 & $3.78 \pm 0.12$ & 4.36 \\
\hline 30.82 & $4.66 \pm 0.15$ & 4.81 & $4.71 \pm 0.15$ & 4.51 & $4.12 \pm 0.13$ & 4.51 & $4.44 \pm 0.14$ & 4.44 & $3.92 \pm 0.13$ & 3.96 & $3.51 \pm 0.11$ & 3.96 \\
\hline 32.06 & $4.97 \pm 0.16$ & 4.80 & $4.54 \pm 0.15$ & 4.43 & $3.94 \pm 0.13$ & 4.43 & $4.26 \pm 0.14$ & 4.26 & $4.01 \pm 0.13$ & 3.89 & $3.32 \pm 0.11$ & 3.89 \\
\hline 35.40 & $4.99 \pm 0.16$ & 4.64 & $4.68 \pm 0.15$ & 4.28 & $4.03 \pm 0.13$ & 4.28 & $4.20 \pm 0.13$ & 4.20 & $3.65 \pm 0.12$ & 3.76 & $3.50 \pm 0.11$ & 3.76 \\
\hline 36.39 & $4.37 \pm 0.14$ & 4.55 & $4.59 \pm 0.15$ & 4.24 & $3.61 \pm 0.12$ & 4.24 & $3.93 \pm 0.13$ & 4.10 & $3.69 \pm 0.12$ & 3.72 & $3.40 \pm 0.11$ & 3.72 \\
\hline 37.26 & $4.43 \pm 0.14$ & 4.61 & $3.98 \pm 0.13$ & 4.21 & $3.15 \pm 0.10$ & 4.21 & $3.32 \pm 0.11$ & 4.14 & $3.73 \pm 0.12$ & 3.70 & $3.29 \pm 0.11$ & 3.70 \\
\hline 43.74 & $3.86 \pm 0.12$ & 4.44 & $3.58 \pm 0.11$ & 4.05 & $3.22 \pm 0.10$ & 4.05 & $3.39 \pm 0.11$ & 3.92 & $3.50 \pm 0.11$ & 3.57 & $3.35 \pm 0.11$ & 3.57 \\
\hline 44.48 & $3.69 \pm 0.12$ & 4.47 & $3.24 \pm 0.10$ & 4.03 & $2.70 \pm 0.09$ & 4.03 & $3.40 \pm 0.11$ & 3.94 & $3.20 \pm 0.10$ & 3.39 & $3.04 \pm 0.10$ & 3.39 \\
\hline 50.38 & $3.67 \pm 0.12$ & 4.29 & $3.39 \pm 0.11$ & 3.94 & $2.83 \pm 0.09$ & 3.94 & $3.37 \pm 0.11$ & 3.93 & $3.33 \pm 0.11$ & 3.48 & $3.16 \pm 0.10$ & 3.48 \\
\hline 51.70 & $3.71 \pm 0.12$ & 4.32 & $2.85 \pm 0.09$ & 3.93 & $2.66 \pm 0.09$ & 3.93 & $3.21 \pm 0.10$ & 3.77 & $3.19 \pm 0.10$ & 3.46 & $2.85 \pm 0.09$ & 3.46 \\
\hline 53.16 & $3.33 \pm 0.11$ & 4.16 & $2.69 \pm 0.09$ & 3.91 & $2.49 \pm 0.08$ & 3.91 & $2.86 \pm 0.09$ & 3.81 & $3.06 \pm 0.10$ & 3.45 & $2.88 \pm 0.09$ & 3.45 \\
\hline 80.99 & $3.53 \pm 0.11$ & 4.01 & $3.02 \pm 0.10$ & 3.78 & $2.59 \pm 0.08$ & 3.78 & $2.78 \pm 0.09$ & 3.63 & $3.40 \pm 0.11$ & 3.34 & $3.21 \pm 0.10$ & 3.34 \\
\hline 276.40 & $4.44 \pm 0.14$ & 4.10 & $4.05 \pm 0.13$ & 3.71 & $3.74 \pm 0.12$ & 3.71 & $3.70 \pm 0.12$ & 3.70 & $3.80 \pm 0.12$ & 3.28 & $3.25 \pm 0.10$ & 3.28 \\
\hline 302.85 & $4.23 \pm 0.14$ & 3.88 & $4.19 \pm 0.13$ & 3.70 & $3.86 \pm 0.12$ & 3.70 & $3.50 \pm 0.11$ & 3.50 & $3.36 \pm 0.11$ & 3.28 & $3.08 \pm 0.10$ & 3.28 \\
\hline 356.01 & $4.13 \pm 0.13$ & 4.13 & $4.12 \pm 0.13$ & 3.71 & $3.78 \pm 0.12$ & 3.71 & $3.39 \pm 0.11$ & 3.73 & $3.28 \pm 0.11$ & 3.28 & $3.28 \pm 0.11$ & 3.28 \\
\hline 383.85 & $4.25 \pm 0.14$ & 3.86 & $3.88 \pm 0.12$ & 3.70 & $3.52 \pm 0.11$ & 3.70 & $3.48 \pm 0.11$ & 3.83 & $3.37 \pm 0.11$ & 3.28 & $3.07 \pm 0.10$ & 3.28 \\
\hline 661.66 & $3.40 \pm 0.11$ & 3.89 & $4.44 \pm 0.14$ & 3.70 & $4.00 \pm 0.13$ & 3.70 & $3.95 \pm 0.13$ & 3.51 & $3.86 \pm 0.12$ & 3.28 & $3.48 \pm 0.11$ & 3.28 \\
\hline
\end{tabular}

can be verified using XCOM software. As seen from Table 5, Compton scattering contributes about $93.69 \%$ to total interaction at $80.99 \mathrm{keV}$, whereas coherent scattering about $3.99 \%$ and photoelectric process only $2.32 \%$ for Glycine. The maximum values of effective atomic number and effective electron density are found in the low energy range, where photoelectric absorption is the main interaction process as seen from Table 3 to 5 . The minimum values of effective atomic number and effective electron density are found at intermediate energies, typically $0.05 \mathrm{MeV}<E<5$ $\mathrm{MeV}$, where Compton scattering is dominant.

In this work, the mass attenuation coefficients of Alanine and Leucine amino acids are investigated for L- and D- isomers. L- and D- amino acids are both optical isomers of each other; they are compounds with the same molecular formula but different spatial arrangements. As seen from Table 2, mass attenuation coefficients of $\mathrm{L}$-amino acids is greater than D- amino acids. Similarly, an effective atomic numbers and electron density of L- amino acids is greater than D- amino acids, as seen from Tables 3 and 4 . These results arise from the molecular structures of different spatial arrangements and can interact with the $\mathrm{X}$ - and $\mathrm{Y}$ ray differently.

In order to study the global reproducibility (any systematic error) of the measurements, five Land D-Alanine samples were prepared and 
Table 4. Effective electron densities $\left(10^{23}\right.$ electron/g) of some amino acids.

\begin{tabular}{|c|c|c|c|c|c|c|c|c|c|c|c|c|}
\hline \multicolumn{13}{|c|}{ Amino acids } \\
\hline $\begin{array}{c}\text { Energy } \\
(\mathrm{keV})\end{array}$ & $\begin{array}{c}\text { Glycine } \\
\text { experimental }\end{array}$ & Theoretical & $\begin{array}{c}\text { L-Alanine } \\
\text { experimental }\end{array}$ & Theoretical & $\begin{array}{c}\text { D-Alanine } \\
\text { experimental }\end{array}$ & Theoretical & $\begin{array}{c}\text { Proline } \\
\text { experimental }\end{array}$ & Theoretical & $\begin{array}{c}\text { L-Leucine } \\
\text { experimental }\end{array}$ & Theoretical & $\begin{array}{c}\text { D-Leucine } \\
\text { experimental }\end{array}$ & Theoretical \\
\hline 8.04 & $5.38 \pm 0.24$ & 5.76 & $6.15 \pm 0.28$ & 6.16 & $5.80 \pm 0.26$ & 6.16 & $6.30 \pm 0.28$ & 6.04 & $7.23 \pm 0.33$ & 6.73 & $6.48 \pm 0.29$ & 6.73 \\
\hline 8.91 & $5.24 \pm 0.24$ & 5.73 & $6.31 \pm 0.28$ & 6.11 & $5.62 \pm 0.25$ & 6.11 & $6.55 \pm 0.29$ & 6.00 & $6.57 \pm 0.30$ & 6.66 & $5.96 \pm 0.27$ & 6.66 \\
\hline 13.37 & $4.94 \pm 0.22$ & 5.40 & $5.36 \pm 0.24$ & 5.67 & $4.81 \pm 0.22$ & 5.67 & $6.54 \pm 0.29$ & 5.54 & $6.38 \pm 0.29$ & 6.04 & $5.45 \pm 0.25$ & 6.04 \\
\hline 14.97 & $4.85 \pm 0.22$ & 5.21 & $5.58 \pm 0.25$ & 5.49 & $4.59 \pm 0.21$ & 5.49 & $5.97 \pm 0.27$ & 5.35 & $5.99 \pm 0.27$ & 5.77 & $5.23 \pm 0.24$ & 5.77 \\
\hline 17.44 & $4.76 \pm 0.21$ & 4.98 & $4.64 \pm 0.21$ & 5.16 & $3.83 \pm 0.17$ & 5.16 & $4.49 \pm 0.20$ & 5.03 & $5.48 \pm 0.25$ & 5.35 & $4.32 \pm 0.19$ & 5.35 \\
\hline 19.63 & $4.76 \pm 0.21$ & 4.76 & $4.57 \pm 0.21$ & 4.89 & $3.81 \pm 0.17$ & 4.89 & $4.46 \pm 0.20$ & 4.76 & $5.21 \pm 0.23$ & 5.02 & $4.43 \pm 0.20$ & 5.02 \\
\hline 22.10 & $4.33 \pm 0.19$ & 4.50 & $4.65 \pm 0.21$ & 4.61 & $3.92 \pm 0.18$ & 4.61 & $4.16 \pm 0.19$ & 4.50 & $4.97 \pm 0.22$ & 4.70 & $4.06 \pm 0.18$ & 4.70 \\
\hline 24.90 & $4.02 \pm 0.18$ & 4.22 & $4.26 \pm 0.19$ & 4.34 & $3.51 \pm 0.16$ & 4.34 & $3.72 \pm 0.17$ & 4.25 & $4.05 \pm 0.18$ & 4.40 & $3.82 \pm 0.17$ & 4.40 \\
\hline 30.82 & $3.74 \pm 0.17$ & 3.86 & $4.14 \pm 0.19$ & 3.96 & $3.62 \pm 0.16$ & 3.96 & $3.95 \pm 0.18$ & 3.88 & $3.96 \pm 0.18$ & 4.00 & $3.55 \pm 0.16$ & 4.00 \\
\hline 32.06 & $3.98 \pm 0.18$ & 3.85 & $3.99 \pm 0.18$ & 3.90 & $3.46 \pm 0.16$ & 3.90 & $3.78 \pm 0.17$ & 3.82 & $4.05 \pm 0.18$ & 3.92 & $3.35 \pm 0.15$ & 3.92 \\
\hline 35.40 & $4.00 \pm 0.18$ & 3.72 & $4.11 \pm 0.18$ & 3.76 & $3.54 \pm 0.16$ & 3.76 & $3.73 \pm 0.17$ & 3.70 & $3.68 \pm 0.17$ & 3.80 & $3.54 \pm 0.16$ & 3.80 \\
\hline 36.39 & $3.51 \pm 0.16$ & 3.65 & $4.03 \pm 0.18$ & 3.73 & $3.17 \pm 0.14$ & 3.73 & $3.50 \pm 0.16$ & 3.67 & $3.73 \pm 0.17$ & 3.76 & $3.43 \pm 0.15$ & 3.76 \\
\hline 37.26 & $3.56 \pm 0.16$ & 3.70 & $3.50 \pm 0.16$ & 3.70 & $2.77 \pm 0.12$ & 3.70 & $2.95 \pm 0.13$ & 3.65 & $3.77 \pm 0.17$ & 3.74 & $3.32 \pm 0.15$ & 3.74 \\
\hline 43.74 & $3.09 \pm 0.14$ & 3.56 & $3.15 \pm 0.14$ & 3.55 & $2.83 \pm 0.13$ & 3.55 & $3.01 \pm 0.14$ & 3.52 & $3.54 \pm 0.16$ & 3.60 & $3.38 \pm 0.15$ & 3.60 \\
\hline 44.48 & $2.96 \pm 0.13$ & 3.58 & $2.85 \pm 0.13$ & 3.54 & $2.37 \pm 0.11$ & 3.54 & $3.03 \pm 0.14$ & 3.50 & $3.23 \pm 0.15$ & 3.43 & $3.07 \pm 0.14$ & 3.43 \\
\hline 50.38 & $2.95 \pm 0.13$ & 3.44 & $2.98 \pm 0.13$ & 3.46 & $2.48 \pm 0.11$ & 3.46 & $2.99 \pm 0.13$ & 3.44 & $3.36 \pm 0.15$ & 3.51 & $3.19 \pm 0.14$ & 3.51 \\
\hline 51.70 & $2.97 \pm 0.13$ & 3.47 & $2.50 \pm 0.11$ & 3.46 & $2.34 \pm 0.11$ & 3.46 & $2.85 \pm 0.13$ & 3.42 & $3.22 \pm 0.14$ & 3.49 & $2.88 \pm 0.13$ & 3.49 \\
\hline 53.16 & $2.67 \pm 0.12$ & 3.34 & $2.36 \pm 0.11$ & 3.44 & $2.19 \pm 0.10$ & 3.44 & $2.54 \pm 0.11$ & 3.42 & $3.09 \pm 0.14$ & 3.49 & $2.90 \pm 0.13$ & 3.49 \\
\hline 80.99 & $2.83 \pm 0.13$ & 3.21 & $2.66 \pm 0.12$ & 3.32 & $2.28 \pm 0.10$ & 3.32 & $2.47 \pm 0.11$ & 3.30 & $3.43 \pm 0.15$ & 3.37 & $3.24 \pm 0.15$ & 3.37 \\
\hline 276.40 & $3.56 \pm 0.16$ & 3.29 & $3.56 \pm 0.16$ & 3.26 & $3.29 \pm 0.15$ & 3.26 & $3.28 \pm 0.15$ & 3.26 & $3.83 \pm 0.17$ & 3.31 & $3.29 \pm 0.15$ & 3.31 \\
\hline 302.85 & $3.39 \pm 0.15$ & 3.11 & $3.68 \pm 0.17$ & 3.25 & $3.39 \pm 0.15$ & 3.25 & $3.11 \pm 0.14$ & 3.25 & $3.40 \pm 0.15$ & 3.31 & $3.11 \pm 0.14$ & 3.31 \\
\hline 356.01 & $3.32 \pm 0.15$ & 3.32 & $3.62 \pm 0.16$ & 3.26 & $3.32 \pm 0.15$ & 3.26 & $3.01 \pm 0.14$ & 3.25 & $3.32 \pm 0.15$ & 3.32 & $3.32 \pm 0.15$ & 3.32 \\
\hline 383.85 & $3.41 \pm 0.15$ & 3.10 & $3.41 \pm 0.15$ & 3.25 & $3.10 \pm 0.14$ & 3.25 & $3.10 \pm 0.14$ & 3.25 & $3.41 \pm 0.15$ & 3.31 & $3.10 \pm 0.14$ & 3.31 \\
\hline 661.66 & $2.73 \pm 0.12$ & 3.12 & $3.90 \pm 0.18$ & 3.25 & $3.51 \pm 0.16$ & 3.25 & $3.51 \pm 0.16$ & 3.24 & $3.90 \pm 0.18$ & 3.31 & $3.51 \pm 0.16$ & 3.31 \\
\hline
\end{tabular}

Table 5. The contributions verified using XCOM to total photon interaction (\%).

\begin{tabular}{|c|c|c|c|c|c|c|c|c|c|c|c|c|}
\hline $\begin{array}{c}\text { Energy } \\
(\mathrm{keV})\end{array}$ & $\begin{array}{c}\text { Glycine } \\
\text { coherent }\end{array}$ & Compton & Photoelectric & $\begin{array}{l}\text { Alanine } \\
\text { coherent }\end{array}$ & Compton & Photoelectric & $\begin{array}{l}\text { Proline } \\
\text { coherent }\end{array}$ & Compton & Photoelectric & $\begin{array}{l}\text { Leucine } \\
\text { coherent }\end{array}$ & Compton & Photoelectric \\
\hline 8.04 & 3.42 & 1.77 & 94.81 & 3.53 & 1.96 & 94.51 & 3.69 & 2.18 & 94.13 & 3.77 & 2.39 & 93.84 \\
\hline 8.91 & 4.09 & 2.49 & 93.42 & 4.22 & 2.76 & 93.02 & 4.43 & 3.06 & 92.51 & 4.51 & 3.35 & 92.14 \\
\hline 13.37 & 7.81 & 9.00 & 83.19 & 8.03 & 9.85 & 82.12 & 8.41 & 10.81 & 80.77 & 8.47 & 11.69 & 79.83 \\
\hline 14.97 & 9.08 & 12.46 & 78.46 & 9.29 & 13.57 & 77.14 & 9.67 & 14.86 & 75.47 & 9.76 & 16.04 & 74.20 \\
\hline 17.44 & 10.76 & 18.79 & 70.45 & 10.96 & 20.37 & 68.66 & 11.33 & 22.01 & 66.67 & 11.36 & 23.58 & 65.07 \\
\hline 19.63 & 11.88 & 25.09 & 63.03 & 12.01 & 26.99 & 61.00 & 12.32 & 28.95 & 58.73 & 12.27 & 30.80 & 56.93 \\
\hline 22.10 & 12.69 & 32.43 & 54.87 & 12.73 & 34.63 & 52.64 & 12.93 & 37.01 & 50.05 & 12.78 & 38.86 & 48.36 \\
\hline
\end{tabular}


Table 5. Contd.

\begin{tabular}{|c|c|c|c|c|c|c|c|c|c|c|c|c|}
\hline 24.90 & 13.07 & 40.99 & 45.94 & 12.99 & 43.38 & 43.63 & 13.05 & 45.67 & 41.28 & 12.82 & 47.79 & 39.39 \\
\hline 30.82 & 12.57 & 56.16 & 31.27 & 12.28 & 58.46 & 29.26 & 12.17 & 60.66 & 27.18 & 11.79 & 62.52 & 25.69 \\
\hline 32.06 & 12.34 & 58.86 & 28.80 & 12.02 & 61.11 & 26.87 & 11.84 & 63.25 & 24.91 & 11.48 & 65.06 & 23.46 \\
\hline 35.40 & 11.55 & 65.35 & 23.10 & 11.19 & 67.42 & 21.39 & 10.96 & 69.35 & 19.68 & 10.55 & 70.99 & 18.46 \\
\hline 36.39 & 11.31 & 67.05 & 21.64 & 10.92 & 69.06 & 20.02 & 10.70 & 70.92 & 18.38 & 10.30 & 72.48 & 17.23 \\
\hline 37.26 & 11.09 & 68.48 & 20.43 & 10.70 & 70.42 & 18.88 & 10.45 & 72.24 & 17.31 & 10.04 & 73.76 & 16.20 \\
\hline 43.74 & 9.45 & 76.92 & 13.63 & 9.08 & 78.39 & 12.53 & 8.75 & 79.89 & 11.36 & 8.41 & 80.98 & 10.60 \\
\hline 44.48 & 9.28 & 77.71 & 13.01 & 8.90 & 79.12 & 11.98 & 8.64 & 80.49 & 10.87 & 8.21 & 81.68 & 10.11 \\
\hline 50.38 & 7.99 & 82.74 & 9.28 & 7.59 & 83.97 & 8.44 & 7.34 & 85.02 & 7.63 & 7.01 & 85.89 & 7.10 \\
\hline 51.70 & 7.69 & 83.70 & 8.61 & 7.36 & 84.75 & 7.89 & 7.11 & 85.78 & 7.11 & 6.73 & 86.68 & 6.59 \\
\hline 53.16 & 7.47 & 84.56 & 7.96 & 7.05 & 85.70 & 7.25 & 6.83 & 86.59 & 6.58 & 6.48 & 87.43 & 6.09 \\
\hline 80.99 & 3.99 & 93.69 & 2.32 & 3.78 & 94.13 & 2.09 & 3.60 & 94.52 & 1.88 & 3.42 & 94.85 & 1.73 \\
\hline 276.40 & 0.54 & 99.40 & 0.06 & 0.51 & 99.43 & 0.06 & 0.48 & 99.47 & 0.05 & 0.46 & 99.50 & 0.05 \\
\hline 302.85 & 0.47 & 99.48 & 0.05 & 0.44 & 99.51 & 0.05 & 0.42 & 99.54 & 0.04 & 0.39 & 99.57 & 0.04 \\
\hline 356.01 & 0.36 & 99.61 & 0.03 & 0.34 & 99.63 & 0.03 & 0.32 & 99.65 & 0.03 & 0.30 & 99.67 & 0.02 \\
\hline 383.85 & 0.32 & 99.65 & 0.03 & 0.30 & 99.67 & 0.02 & 0.29 & 99.69 & 0.02 & 0.27 & 99.71 & 0.02 \\
\hline 661.66 & 0.13 & 99.86 & 0.01 & 0.13 & 99.87 & 0.01 & 0.12 & 99.87 & 0.01 & 0.11 & 99.88 & 0.01 \\
\hline
\end{tabular}

measured under the same experimental conditions. Calculated relative standard deviations (RSD) are given in Table 6. Keeping in mind that the values obtained following this procedure also include the uncertainty due to sample preparation, and instrument and counting statistics, the RSD values are satisfactory. Additionally, one of the Land D- Alanine samples was measured 5 times and the RSD associated was also calculated Note that the RSD values obtained using the five samples prepared under the same conditions are higher than those obtained for the same sample measured 5 times. Because, for the same sample measured 5 times, the RSD is very small, it is concluded that the spectrometry sensitivity was very good.

Manual errors might occur when the samples were weighed. However, from the data obtained, it could be deduced that the uncertainty introduced by sample preparation is acceptable.
The overall error in the experimental parameters is the sum of the uncertainties in different factors, namely, the evaluation of peak areas $(2.10$ to $7.00 \%)$, target mass thickness (1.50 to $3.90 \%)$ and statistical error $(<1.00 \%)$. Total errors affecting the experimental parameters are calculated between 2.77 and $8.08 \%$. The errors in the evaluation of the areas under the $\mathrm{X}$ - and $\mathrm{y}$ - ray peaks have two main error sources, that is, the errors in the elimination of the background and in the peak fitting procedures.

\section{Conclusions}

We reported new data on mass attenuation coefficient, effective atomic number and effective electron density in the energy range of 8.04 to $661.66 \mathrm{keV}$. Values of mass attenuation coefficient, effective atomic number and effective electron density depend on photon energy and chemical content of amino acids. The sensitive experiments would be required to study the effect of chemical bonding on physical parameters of amino acids.

The photon interaction parameters in low $Z$ composite materials would be investigated to confirm the applicability of mixture rule at different energies. Mass attenuation coefficient, effective atomic number and effective electron density would be determined for L- and D- isomers of other amino acids. The present calculations of mass attenuation coefficient, effective atomic number and effective electron density have thrown new light on the underlying radiation physics and will hope fully be useful in medical and biological application. Also, to the best knowledge of the authors, these data are the first of their kind at these energies. 
Table 6. Relative standard deviations (RSD) of L- and D-Alanine.

\begin{tabular}{ccccc}
\hline & \multicolumn{2}{c}{ L-Alanine } & \multicolumn{2}{c}{ D-Alanine } \\
\cline { 2 - 5 } $\begin{array}{c}\text { X- and y- ray } \\
\text { peaks (keV) }\end{array}$ & $\begin{array}{c}\text { 5 samples/ } \\
\text { 1 measurement } \\
\text { RSD (\%) }\end{array}$ & $\begin{array}{c}\text { 1 sample/ } \\
\text { 5 measurement } \\
\text { RSD (\%) }\end{array}$ & $\begin{array}{c}\text { 5 samples/ } \\
\text { 1 measurement } \\
\text { RSD (\%) }\end{array}$ & $\begin{array}{c}\text { 1 sample/ } \\
\text { 5 measurement } \\
\text { RSD (\%) }\end{array}$ \\
\hline 8.04 & 0.95 & 0.28 & 0.86 & 0.24 \\
8.91 & 0.95 & 0.30 & 0.87 & 0.23 \\
13.37 & 0.93 & 0.28 & 0.93 & 0.28 \\
14.97 & 0.90 & 0.25 & 0.80 & 0.27 \\
17.44 & 0.77 & 0.30 & 0.82 & 0.30 \\
19.63 & 0.89 & 0.32 & 0.90 & 0.31 \\
22.10 & 0.92 & 0.32 & 0.84 & 0.24 \\
24.90 & 0.95 & 0.31 & 0.95 & 0.32 \\
30.82 & 0.85 & 0.24 & 0.82 & 0.24 \\
32.06 & 0.78 & 0.32 & 0.86 & 0.28 \\
35.40 & 0.91 & 0.22 & 0.91 & 0.22 \\
36.39 & 0.86 & 0.23 & 0.76 & 0.23 \\
37.26 & 0.82 & 0.22 & 0.92 & 0.25 \\
43.74 & 0.94 & 0.23 & 0.84 & 0.23 \\
44.48 & 0.84 & 0.23 & 0.86 & 0.31 \\
50.38 & 0.85 & 0.26 & 0.75 & 0.26 \\
51.70 & 0.81 & 0.32 & 0.89 & 0.28 \\
53.16 & 0.79 & 0.22 & 0.79 & 0.35 \\
80.99 & 0.82 & 0.31 & 0.72 & 0.29 \\
276.40 & 0.72 & 0.26 & 0.72 & 0.32 \\
302.85 & 0.86 & 0.22 & 0.86 & 0.28 \\
356.01 & 0.92 & 0.23 & 0.76 & 0.33 \\
383.85 & 0.79 & 0.20 & 0.74 & 0.35 \\
661.66 & 0.75 & 0.22 & 0.85 & 0.32 \\
\hline & & & &
\end{tabular}

\section{REFERENCES}

Akkurt I (2007). Effective atomic numbers for Fe-Mn alloy using transmission experiment. Chin. Phys. Lett. 24(10):2812-2814.

Akkurt I (2009). Effective atomic and electron numbers of some steels at different energies. Ann. Nucl. Energy 36(11-12):1702-1705.

Gerward L, Guilbert N, Jensen KB, Levring (2001). X-ray absorption in matter reengineering XCOM. Radiat. Phys. Chem. 60:23-24.

Gowda S, Krishnaveni S, Gowda R (2005). Studies on effective atomic numbers and electron densities in amino acids and sugars in the energy range 30-1333 keV. Nucl. Inst. Meth. B 239:361-369.

Hine GJ (1952). The effective atomic numbers of materials for various gamma interactions. Phys. Rev. 85:725-728.

Hiremath SS, Chikkur GC (1993). Computation of effective atomic number of some hydrocarbons containing $\mathrm{H}, \mathrm{C}$ and $\mathrm{O}$ atoms in the energy range 54-1333 keV. Indian J. Pure Appl. Phys. 31:855-860.

Jackson DF, Hawkes DJ (1981). X-ray attenuation coefficients of elements and mixtures. Phys. Reports 70:169-233.

Kerur BR, Thontadarya SR, Hanumaiah B (1991). A novel method for the determination of $x$-ray mass attenuation coefficients. Appl. Radiat. Isot. 42:571-576.

Keszthelyi L, Vincze I (1975). Absorption of circularly polarized gamma radiation in L- and D-amino acids. Radiat. Environ. Biophys. 12:181185.

Kumar A, Singh S, Mudahar GS, Thind KS (2006). Molar extinction coefficients of some commonly used solvents. Radiat. Phys. Chem. 75:737-740

Manjunathaguru V, Umesh TK (2006). Effective atomic numbers and electron densities of some biologically important compounds containing $\mathrm{H}, \mathrm{C}, \mathrm{N}$ and $\mathrm{O}$ in the energy range $145-1330 \mathrm{keV}$. J. Phys. B. At. Mol. Opt. Phys. 39:3969-3981.

Manjunathaguru V, Umesh TK (2009). Simple parametrization of photon mass energy absorption coefficients of $\mathrm{H}, \mathrm{C}, \mathrm{N}$ and $\mathrm{O}$ based samples of biological interest in the energy range $200-1500 \mathrm{keV}$. Pramana. J. Phys. 72:375-387.

Manohara SR, Hanagodimath SM, Gerward L (2008a). Energy dependence of effective atomic numbers for photon energy absorption and photon interaction: Studies of some biological molecules in the energy range $1 \mathrm{keV}-20 \mathrm{MeV}$. Med. Phys. 35:388402.

Manohara SR, Hanagodimath SM, Thind KS, Gerward L (2008b). On the effective atomic number and electron density: A comprehensive set of formulas for all types of materials and energies above $1 \mathrm{keV}$. Nucl. Instr. Meth. B. 266:3906-3912.

Monohara SR, Hanagodimath SM (2007a). Studies on effective atomic numbers and electron densities of essential amino acids in the energy range $1 \mathrm{keV}-100 \mathrm{GeV}$. Nucl. Instr. Meth. B 258:321-328.

Monohara SR, Hanagodimath SM (2007b). Effective atomic numbers for photon energy absorption of essential amino acids in the energy range $1 \mathrm{keV}$ to $20 \mathrm{MeV}$. Nucl. Instr. Meth. B 264:9-14.

Özyol H (1994). Study of effective atomic numbers in the alloy for gamma ray interactions in the energy region 100-662 keV. Indian J. Pure Appl. Phys. 6:609-613.

Rao BVT, Raju MLN, Rao BM, Narasimham KL, Parthasaradhi K (1985). Interaction of low energy photons with biological samples and effective atomic number. Med. Phys. 12:745-748.

Sharanabasappa KBR, Anilkumar S, Hanumaiah B (2010). Determination of $x$-ray mass attenuation coefficients using HPGe 
detector. Appl. Radiat. Isot. 68:76-83.

Singh K, Sandhuand GK, Lark BS (2004). Molar extinction coefficients of solutions of some organic compounds. Pramana. J. Phys. 62:1139-1145.

Singh T, Kaur U, Tandon S, Singh PS (2010). A study of photon interaction and photon energy absorption effective atomic numbers for some amino acids. Nucl. Sci. Eng. 165: 240-244.
Voet D, Voet JG (1995). Biochemistry. John Wiley and Sons London. pp. 56-81.

Yang NC, Leichner PK, Hawkins WG (1987). Effective atomic number for low energy total photon interactions in human tissue. Med. Phys. 14:759-766. 